\title{
Improved Waveform Design for Target Recognition with Multiple Transmissions
}

\author{
Ric Romero and Nathan A. Goodman \\ Electrical and Computer Engineering \\ University of Arizona \\ Tucson, AZ \\ \{ricr@email,goodman@ece\}.arizona.edu
}

\begin{abstract}
This paper presents a matched waveform technique for target class identification, i.e., a multiple hypotheses testing (MHT) framework. This technique is shown to improve classification performance of SNR-based matched waveforms derived from a probability-weighted spectral variance (PWSV) approach. The technique, which allows for real-time adaptive waveform transmission, is also shown to be computationally efficient.
\end{abstract}

\section{INTRODUCTION}

Optimum waveform design for target detection and/or target recognition has received a fair amount of attention of late. For example, matched waveform design for detecting a known target in additive Gaussian noise was addressed via the SNR criterion in [1]. An early attempt to the problem of matched waveform design for a deterministic extended target in signaldependent interference using the SNR criterion was proposed in [2], where an algorithm was developed to form a finiteduration transmit waveform matched to a known target in the presence of signal-dependent clutter. From the frequency domain approach, the SNR-based optimum matched waveform for a known target in signal-dependent interference was derived in [3]. For estimating the parameters of a random target, Bell [1] used the information-theoretic metric of mutual information (MI) in designing a transmit waveform matched to a Gaussian ensemble. We extended this information-theoretic approach for waveform design in signal-dependent clutter in [4]. Waveform design for detecting a Gaussian-distributed point target in Gaussian clutter was addressed by Kay in [5].

In one of our earlier works [6], SNR- and MI-based waveforms were implemented in a closed-loop radar system performing target recognition from a set of possible alternatives, i.e., a multiple hypotheses testing (MHT) problem. However, waveform design strategies presented in the literature (including our own derivations [3, 4]) are matched only to a single deterministic target or stochastic target class. Therefore, to apply the waveforms to a multi-hypotheses recognition problem, we proposed to use a probability-weighted spectral variance (PWSV) over the hypothesis ensemble [6]. This PWSV was substituted for the target characteristics in the waveform design equations, and effectively accounted for all target hypotheses. As the hypothesis probabilities change with each transmission/observation, the PWSV changes, and the waveform adapts.
Our results obtained from the PWSV approach have been good, but here we report an improved technique. In the new technique, we calculate the MI- or SNR-based matched waveform for each hypothesis, then combine the waveforms in proportion to the hypothesis probabilities. The new technique significantly improves the SNR-based approach for the multihypotheses problem and provides significant computational savings when waveforms are to be adapted in real time. The focus in this paper is on the class discrimination problem where each hypothesis is in fact a class of target realizations.

This paper is organized as follows. Section II summarizes MI and SNR matched waveform designs for a random target or target class. Section III discusses closed-loop radar system for target discrimination utilizing the MI and SNR matched waveforms. Section IV summarizes the the previously reported transmission strategy and presents the new one. We present results in Section V and our concluding remarks are in Section VI.

\section{MI AND SNR MATCHED WAVEFORMS}

Aside from the MI-based waveform design in [4], we also derived and applied matched waveform design via the SNR criterion for a stochastic target in [3]. Here, we summarize the MI and SNR matched waveform designs in both signal-dependent interference and signal-independent noise. Referring to Fig.1, let $\boldsymbol{h}(t)$ or $\boldsymbol{H}(f)$ represent the zero-mean complex-valued stochastic Gaussian target with duration $T_{h}$, $\boldsymbol{c}(t)$ be the zero-mean complex-valued stochastic Gaussian clutter response described by the PSD $S_{c c}(f), x(t)$ be the $T$ duration transmit wavform, $\boldsymbol{n}(t)$ be the receiver noise with PSD $S_{n n}(f)$, and $\boldsymbol{y}(t)$ be the output measurement. Then, the MI between a Gaussian target ensemble and the received signal is given by

$$
\begin{aligned}
& I(\boldsymbol{y}(t) ; \boldsymbol{h}(t) \mid x(t)) \\
& \quad=T_{z} \int_{W} \ln \left[1+\frac{|X(f)|^{2} \sigma_{H}^{2}(f)}{T_{z}\left\{S_{n n}(f)+|X(f)|^{2} S_{c c}(f)\right\}}\right] d f,
\end{aligned}
$$

where $T_{z}=T+T_{h}$ is the duration of the convolved transmit waveform and target. The term $\sigma_{H}^{2}(f)$ is the energy spectral variance [1] of the target ensemble given by

$$
\sigma_{H}^{2}(f)=\mathrm{E}\left[\left|\boldsymbol{H}(f)-\mu_{H}(f)\right|^{2}\right]
$$


and $\mu_{H}(f)$ is the spectral mean of the target process defined by

$$
\mu_{H}(f)=E[\boldsymbol{H}(f)]
$$

Using the transmit energy constraint given by

$$
\int_{W}|X(f)|^{2} d f \leq E_{x}
$$

the transmit waveform spectrum that maximizes MI by Lagrangian multiplier technique is given by

$$
|X(f)|^{2}=\max \left[0,-R(f)+\sqrt{R^{2}(f)+S(f)(A-D(f))}\right],
$$

where

$$
\begin{gathered}
D(f)=\frac{T_{z} S_{n n}(f)}{\sigma_{H}^{2}(f)} \\
R(f)=\frac{S_{n n}(f)\left(2 S_{c c}(f)+T_{z}^{-1} \sigma_{H}^{2}(f)\right)}{2 S_{c c}(f)\left(S_{c c}(f)+T_{z}^{-1} \sigma_{H}^{2}(f)\right)}
\end{gathered}
$$

and

$$
S(f)=\frac{S_{n n}(f) T_{z}^{-1} \sigma_{H}^{2}(f)}{S_{c c}(f)\left(S_{c c}(f)+T_{z}^{-1} \sigma_{H}^{2}(f)\right)} .
$$

The constant $A$ is determined by the energy constraint

$\int_{W} \max \left[0,-R(f)+\sqrt{R^{2}(f)+S(f)(A-D(f))}\right] d f \leq E_{x}$.

Formation of the optimum waveform requires numerical search for $A$. For the non-clutter case, the optimum waveform spectrum is given by

$$
|X(f)|^{2}=\max \left[0, A-\frac{T_{z} S_{n n}(f)}{\sigma_{H}^{2}(f)}\right],
$$

where $A$ is again searched such that the energy constraint is now dictated by

$$
\int_{W} \max \left[0, A-\frac{T_{z} S_{n n}(f)}{\sigma_{H}^{2}(f)}\right] d f \leq E_{x} .
$$

The SNR criterion has been derived in [3] and is given by

$$
S N R \simeq \int_{W} \frac{\sigma_{H}^{2}(f)|X(f)|^{2}}{S_{c c}(f)|X(f)|^{2}+S_{n n}(f)} d f .
$$

The optimum transmit waveform is given by

$$
|X(f)|^{2}=\max [0, B(f)(A-D(f))]
$$

where $B(f)$ and $D(f)$ are described by

$$
B(f)=\frac{\sqrt{\sigma_{H}^{2}(f) S_{n n}(f)}}{S_{c c}(f)}
$$

and

$$
D(f)=\sqrt{\frac{S_{n n}(f)}{\sigma_{H}^{2}(f)}}
$$

and $A$ is determined such that the energy constraint is now

$$
\int_{W} \max [0, B(f)(A-D(f))] d f \leq E_{x} .
$$

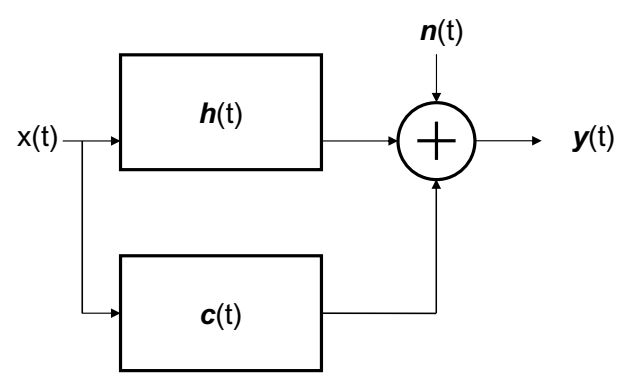

Fig. 1. Stochastic extended target in signal-dependent interference.

For the non-clutter case, it can be shown that the finiteduration, energy-constrained $x(t)$ that maximizes SNR is given by the primary eigen-function of $[1,3]$

$$
\lambda_{\max } \check{x}(t)=\int_{-T / 2}^{T / 2} \check{x}(\tau) R_{h}(t-\tau) d \tau,
$$

where the kernel $R_{h}(t)$ is

$$
R_{h}(t)=\int_{-\infty}^{\infty} \frac{\sigma_{H}^{2}(f)}{S_{n n}(f)} e^{j 2 \pi f t} d f .
$$

\section{TARget Recognition Closed-Loop Strategy}

In [6], we proposed a closed-loop radar system for a target recognition problem. It is enough to mention that we will utilize such a system with multiple transmissions. Unlike the system in [6], where the sequential hypothesis testing (SHT) is used to terminate an experiment, here we will fix the number of transmissions (indeed, many practical systems operate in this fashion) and choose the the most likely hypothesis at the last probability update in making an identification.

Consider the multiple target testing problem where the data model for the $i^{t h}$ hypothesis is

$$
H_{i}: \boldsymbol{y}(t)=x(t) * \boldsymbol{h}_{i}(t)+x(t) * \boldsymbol{c}(t)+\boldsymbol{n}(t)
$$

where $\boldsymbol{h}_{i}(t)$ represents one of the $M$ possible random targets. For the non-clutter case, we simply set $\boldsymbol{c}(t)=0$. In discretetime implementation, the model above translates to

$$
H_{i}: \mathbf{y}=\mathbf{X}\left(\mathbf{h}_{i}+\mathbf{c}\right)+\mathbf{n}
$$

where $\mathbf{X}$ is the transmit-waveform convolution matrix [7], $\mathbf{h}_{i}$ is the length- $L_{h}$ random target vector under the $i^{\text {th }}$ hypothesis, $\mathbf{c}$ is the random clutter vector, $\mathbf{n}$ is the signal-independent noise, and $\mathbf{y}$ is the length- $L$ received vector. Let $\sigma_{i}^{2}(f)$ be the spectral variance that describes the target under the $i^{\text {th }}$ class. While we presented matched illumination for a target class in the previous section, the present problem is classification from an ensemble of classes. Thus, a waveform design strategy is needed for this MHT framework. Utilizing the notion of spectral variance [1], we previously defined the probability 
weighted spectral variance (PWSV) for the whole ensemble (over all hypotheses) to be

$$
\sigma_{T}^{2}(f)=\sum_{i=1}^{M} \operatorname{Pr}\left(H_{i}\right) \sigma_{i}^{2}(f)-\left|\sum_{i=1}^{M} \operatorname{Pr}\left(H_{i}\right) \sqrt{\sigma_{i}^{2}(f)}\right|^{2},
$$

where $\operatorname{Pr}\left(H_{i}\right)$ is the probability that the $i^{t h}$ hypothesis is true. Equation (19) may be considered as the effective PSD of the ensemble. This effective PSD can be substituted into waveform design strategies optimizing either SNR or MI.

For a closed-loop radar performing target class discrimination in a Bayesian framework, the PWSV is clearly convenient since a probability update is easily accommodated by (19). For a discrete-time implementation of the closed-loop radar, the probability update rule for the $i^{t h}$ target for $(k+1)^{t h}$ transmission is given by

$$
P_{i}^{k+1}=\beta p_{i}\left(\mathbf{y}_{1}, \mathbf{y}_{2}, \ldots, \mathbf{y}_{k}\right) P_{i}^{k},
$$

where $p_{i}\left(\mathbf{y}_{1}, \mathbf{y}_{2}, \ldots, \mathbf{y}_{k}\right)$ is the $p d f$ of the measurement after the $k^{\text {th }}$ transmission and $\beta$ ensures unity probability over the classes at each iteration. The expression for $p\left(\mathbf{y}_{1}, \mathbf{y}_{2}, \ldots, \mathbf{y}_{k}\right)$ may take different forms depending if the measurements are correlated or not. Since the $p d f$ is dependent on the scenario being considered, we will present such a $p d f$ for the example considered in the Results section.

\section{The PWSV AND PWE TRANSMISSION TECHNIQUES}

Looking at the top half of Fig. 2, we refer to the transmit waveform approach previously reported as the PWSV transmission technique. At each iteration, there are two major operations in which the waveform is formed. The first is the calculation of the PWSV. The probability updates are used in a set of gain scaling of the individual variances and a set of gain scaling of the individual root-variances. In addition, an absolute value, a negate operation and a couple of summations are finally used to form the PWSV. The second operation, which uses the PWSV as an input, is an algorithm which effectively searches for the optimum waveform. For example, the well known bisection algorithm may be used to generate a waterfilling waveform. Unfortunately, the number of operations it takes to do a search depends on the current PWSV and the energy constraint. Furthermore, it also depends on the assumption for the initial boundaries of $A$.

Now, we introduce a simpler technique that also proves to be more effective. Instead of finding a PWSV prior to forming a waveform, the new approach, which is termed probability weighted energy (PWE) technique, forms individualized waveform spectra, $\left|X_{i}^{\text {opt }}(f)\right|^{2}$, which are optimum under SNR or MI for each hypothesis. Then, the individual waveform spectra are weighted by the probability updates in order to meet an overall energy constraint. Thus, the waveform is

$$
\left|X_{T}^{2}(f)\right|^{2}=\sum_{i=1}^{M} \operatorname{Pr}\left(H_{i}\right)\left|X_{i}^{o p t}(f)\right|^{2} .
$$

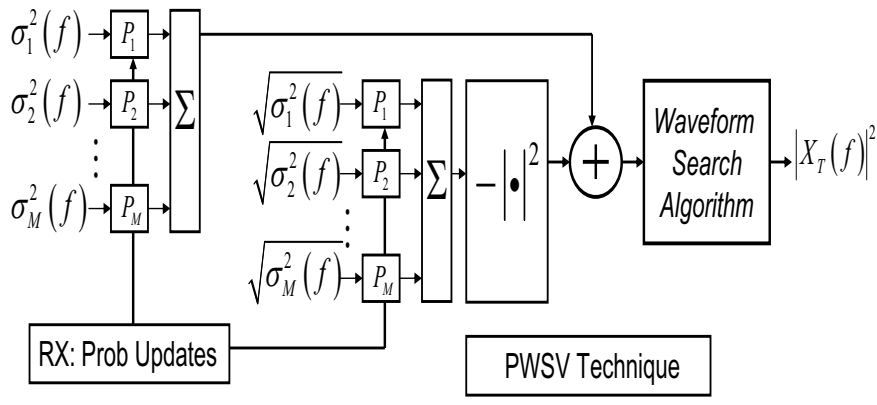

PWE Technique



Fig. 2. Two closed-loop multiple transmission techniques for one iteration top: PWSV technique, bottom: PWE technique

That is, the transmit waveform spectrum is effectively the sum of the individual matched waveforms scaled by their corresponding update probabilities. To appreciate the computational efficiency of the technique, we refer to the bottom half of Fig. 2, where it is clear that the formation of transmit waveform involves only gain scaling of pre-calculated spectral coefficients and one summation.

In summary, the PWE transmission technique eliminates one set of gain scaling, an absolute value, a negation, and a summation for each transmission. More importantly, it eliminates the waveform search algorithm, which potentially causes two problems for a system utilizing multiple transmissions. The first is the extra use of computational resource, which could be taxing for a radar forming transmit waveform in a closedloop fashion. The second is latency. The search algorithm, which could easily take a substantial number of iterations to converge, adds valuable time to the formation of a waveform, which may not be acceptable to a system employing real-time adaptive transmission.

\section{Results}

Here we set up a target class recognition problem where the MHT problem consists of four hypotheses, whose target spectral variances are shown in Fig. 3. As mentioned earlier, the $p d f$ of received vectors is needed in closed-loop operation such that a probability update may be calculated. While different scenarios are possible, we consider a very likely scenario where both the target and clutter realizations do not change within the entire duration of the interrogation. Then, the $p d f$ of the received signal at $k^{t h}$ iteration under the $i^{t h}$ 
target hypothesis is given by

$$
\begin{aligned}
& p_{i}\left(\mathbf{y}_{1}, \ldots, \mathbf{y}_{k}\right) \\
& =\frac{\left|\mathbf{Q}^{-1}\right|}{\left|\mathbf{K}_{\mathbf{t}}\right| \pi^{L k}\left|\mathbf{K}_{\mathbf{N}}\right|^{k}} \exp \left[-\sum_{j=1}^{k} \mathbf{y}_{k}^{\mathrm{H}} \mathbf{K}_{\mathbf{N}}{ }^{-1} \mathbf{y}_{k}\right] \\
& \quad \times \exp \left[\left[\sum_{j=1}^{k} \mathbf{X}_{k}^{\mathrm{H}} \mathbf{K}_{\mathbf{N}}{ }^{-1} \mathbf{y}_{k}\right]^{H} \mathbf{Q}^{-1} \sum_{j=1}^{k} \mathbf{X}_{k}^{\mathrm{H}} \mathbf{K}_{\mathbf{N}}{ }^{-1} \mathbf{y}_{k}\right],
\end{aligned}
$$

where $\mathbf{K}_{\mathbf{t}}$ and $\mathbf{Q}$ are defined by

$$
\begin{gathered}
\mathbf{K}_{\mathbf{t}}=\mathbf{K}_{\mathbf{h} i}+\mathbf{K}_{\mathbf{c}} \\
\mathbf{Q}=\mathbf{K}_{\mathbf{t}}^{-1}+\sum_{j=1}^{k} \mathbf{X}_{k}^{\mathrm{H}} \mathbf{K}_{\mathbf{N}}{ }^{-1} \mathbf{X}_{k},
\end{gathered}
$$

$\mathbf{K}_{\mathbf{h} i}$ is the target covariance matrix of target hypothesis vector $\mathbf{h}_{i}, \mathbf{K}_{\mathbf{c}}$ is the clutter covariance matrix of the clutter vector $\mathbf{c}$, and $\mathbf{K}_{\mathbf{N}}$ is the noise covariance. Here, we model the signalindependent noise as AWGN. To illustrate the improvement of the new waveform technique, we do not need to consider the case where clutter is present. Thus, we set $\mathbf{c}=0$. Then, the $p d f$ of resulting measurements reduces to

$$
\begin{aligned}
& p_{i}\left(\mathbf{y}_{1}, \ldots, \mathbf{y}_{k}\right) \\
& =\frac{\left|\mathbf{Q}^{-1}\right|}{\left|\mathbf{K}_{\mathbf{h} i}\right| \pi^{L k}\left(\sigma_{n}^{2}\right)^{L k}} \exp \left[-\left(\sigma_{n}^{2}\right)^{-1} \sum_{j=1}^{k} \mathbf{y}_{k}^{\mathrm{H}} \mathbf{y}_{k}\right] \\
& \quad \times \exp \left[\left(\sigma_{n}^{4}\right)^{-1}\left[\sum_{j=1}^{k} \mathbf{X}_{k}^{\mathrm{H}} \mathbf{y}_{k}\right]^{H} \mathbf{Q}^{-1} \sum_{j=1}^{k} \mathbf{X}_{k}^{\mathrm{H}} \mathbf{y}_{k}\right]
\end{aligned}
$$

where $\left(\sigma_{n}^{2}\right)^{-1}$ replaces $\mathbf{K}_{\mathbf{N}}{ }^{-1}$ in (23).

We report on the multi-hypotheses classification performance of five different waveforms. We include results for both a single transmission, and for adaptive multiple transmission experiments. For the multiple transmission, we fixed the number of transmissions to 10 . To extract waveform classification performance over different energy constraints, we used Monte Carlo simulation, where we generated 1000 target realizations randomly chosen from the 4 hypotheses. For each target realization, we generated 10 noise realizations to average over. The waveforms are: a) spectral variance derived SNR waveform (SNR-PWSV), b) the new probability-weighted energy SNR waveform (SNR-PWE), c) spectral variance derived MI waveform (MI-PWSV), d) probability-weighted energy MI waveform (MI-PWE), and e) an unmatched wideband pulsed waveform (WB). Referring to Fig. 4, which depicts a single transmission experiment, we notice the vast improvement of the new technique (SNR-PWE) over the previous technique (SNR-PWSV). In fact, it seems to be the best performer compared to all of the waveforms except at high energy. Interestingly, in this case of single transmission experiment, the new technique applied to MI has lower performance. However, when evaluating classification performance with multiple
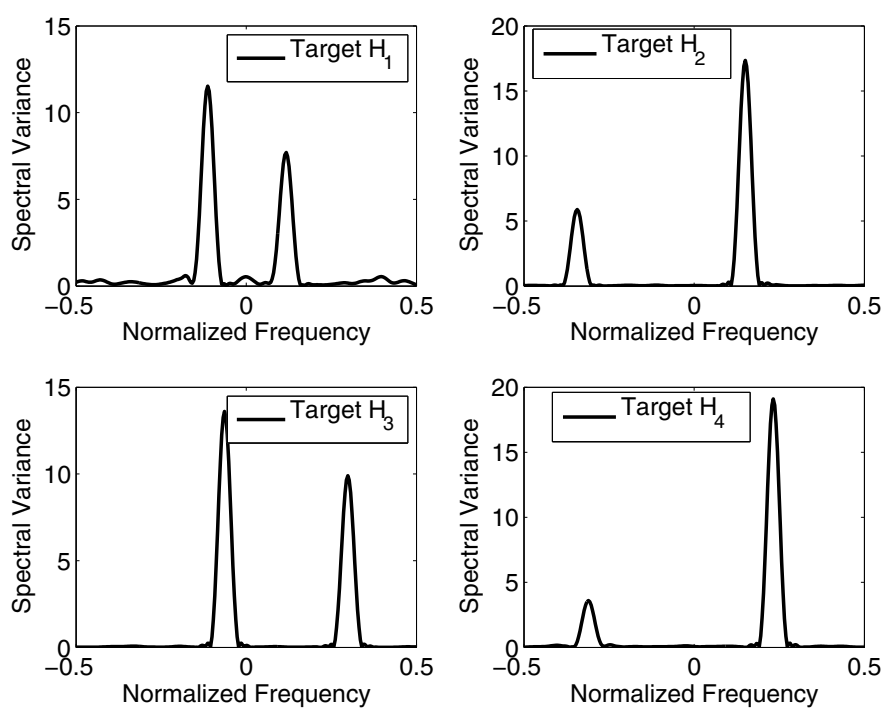

Fig. 3. Target Spectral Variances

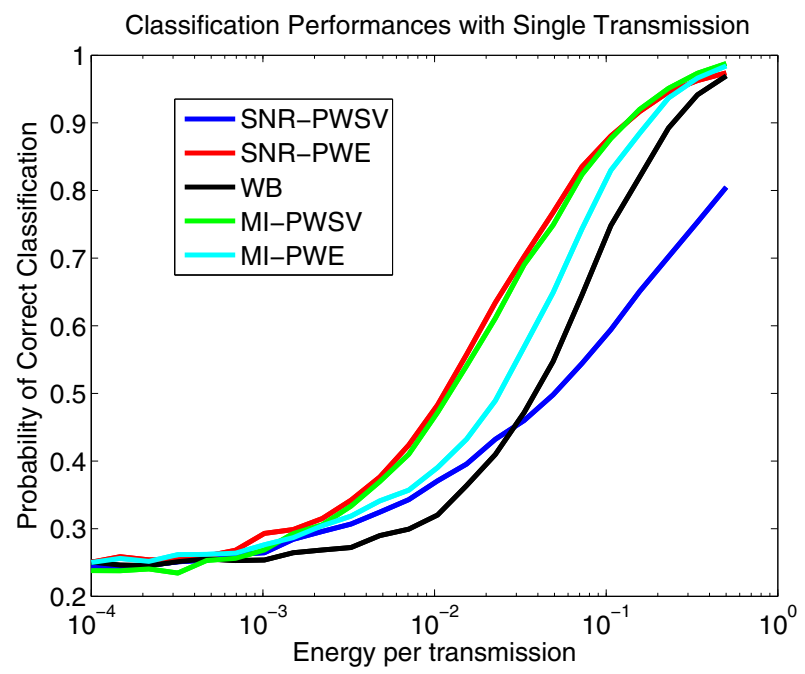

Fig. 4. Classification performances with single transmission

transmissions, the new technique performs very well under both SNR- and MI-based design metrics. Closed-loop results for classification with 10 transmissions is shown in Figure 5, where again the SNR-PWE is the best performer except at high energy. Although the performance benefit of the MIPWE over the previous MI-PWSV waveform is minor, when the computational benefits are considered, the new technique is a nice improvement.

\section{CONCLUSION}

For a closed-loop radar performing class discrimination from an ensemble of Gaussian target classes and employing multiple transmissions, we presented two techniques in which to form transmit waveforms in real time. We had shown that the PWSV technique, previously reported in [6], required both calculation of an effective PSD and a costly search algorithm to generate a transmit waveform for each iteration. Here, we 


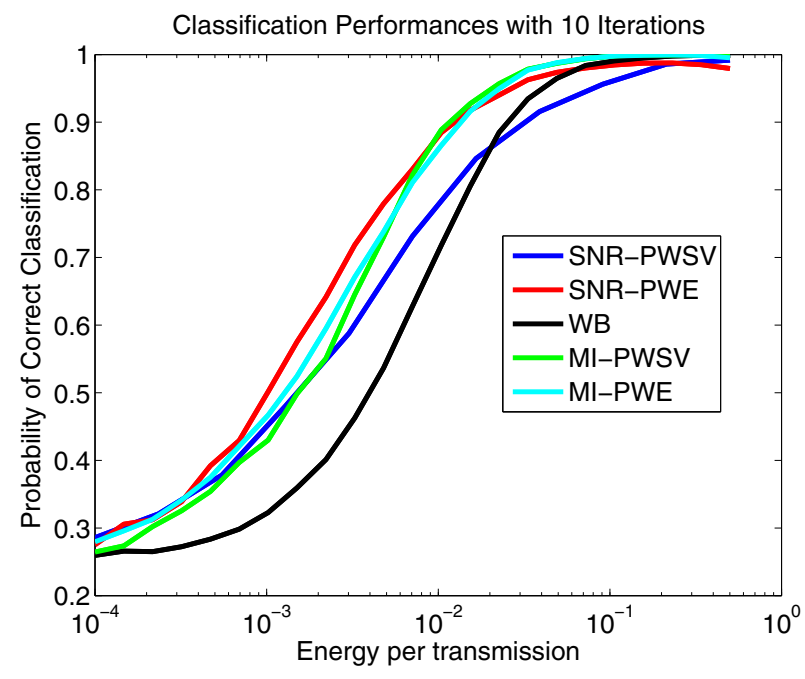

Fig. 5. Classification performances with 10 transmissions

reported a simpler technique called PWE, which pre-calculated individual SNR or MI matched waveforms for each target class. Then it simply utilized the probability updates as gain scalers for the the individual matched waveforms and summed the results to form the transmit waveform. More importantly, it eliminated the need to use a search algorithm, which could cause both computational resource and latency issues for a radar generating adaptive transmissions. As for classification performance, the PWE technique applied to SNR (SNR-PWE) was shown to be the best performer for both single and multitransmission experiments except for high-energy.

\section{REFERENCES}

[1] M. R. Bell, "Information theory and radar waveform design," IEEE Trans. Inform. Theory, vol. 39, no. 5, pp. 1578-1597, Sep. 1993.

[2] S. U. Pillai, H. S. Oh, D. C. Youla, and J. R. Guerci, "Optimum transmitreceiver design in the presence of signal-dependent interference and channel noise," IEEE Trans. Inform. Theory, vol. 46, no. 2, pp. 577-584, Mar. 2000.

[3] R. Romero, J. Bae, and N. Goodman "Theory and application of SNRand MI-based matched illumination waveforms ," submitted to IEEE Trans. Aero. \& Syst.

[4] R. Romero and N. Goodman, "Information-theoretic matched waveform in signal dependent interference," in Proc. IEEE 2008 Radar Conf., Rome, Italy, May 26-30, 2008

[5] S. Kay, "Optimal signal design for detection of Gaussian point targets in stationary Gaussian clutter/reverberation ," IEEE J. Sel. Topics in Sig. Proc. Mag., vol. 1, no. 1, pp. 31-41, Jun. 2007.

[6] N. Goodman, P. Venkata, and M. Neifeld, "Adaptive waveform design and sequential hypothesis testing for target recognition with active sensors, IEEE J. Sel. Topics in Sig. Proc. Mag., vol. 1, no. 1, pp. 105-113, Jun. 2007.

[7] D. A. Garren, M. K. Osborn, A. C. Odom, J. S. Goldstein, S. U. Pillai, and J. R. Guerci, "Enhanced target detection and identification via optimised radar transmission pulse shape," Proc. IEEE, vol. 148, no. 3, pp. 130-138, Jun. 2001. 